\title{
The effects of individual differences and task difficulty on inattentional blindness
}

\author{
Daniel J. Simons and Melinda S. Jensen \\ University of Illinois at Urbana-Champaign, Urbana, Illinois
}

\begin{abstract}
Most studies of inattentional blindness - the failure to notice an unexpected object when attention is focused elsewhere - have focused on one critical trial. For that trial, noticing the unexpected object might be a result of random variability, so that any given individual would be equally likely to notice the unexpected object. On the other hand, individual differences in the ability to perform the primary task might make noticing more likely for some individuals than for others. Increasing the difficulty of the primary task has been shown to decrease noticing rates for both brief static displays (Cartwright-Finch \& Lavie, 2007) and dynamic monitoring tasks (Simons \& Chabris, 1999). However, those studies did not explore whether individual differences in noticing arise from differences in the ability to perform the primary task. For our Experiment 1, we used a staircase procedure to equate primary task performance across individuals in a dynamic inattentional blindness task and found that the demands of the primary task affected noticing rates when individual differences in accuracy were minimized. In Experiment 2, we found that individual differences in primary task performance did not predict noticing of an unexpected object. Together, these findings suggest that although the demands of the primary task do affect inattentional blindness rates, individual differences in the ability to meet those demands do not.
\end{abstract}

Inattentional blindness is the striking failure to notice a fully visible but unexpected object when attention is otherwise engaged (Mack \& Rock, 1998; Simons, 2007). In a typical inattentional blindness task, observers perform an attention-demanding task for several trials, and on a critical trial, an additional object appears unexpectedly. Immediately after that trial, observers are asked to report whether or not they noticed the unexpected object. Across a wide range of displays and tasks, many observers fail to report the unexpected objects, even when the objects are distinctive and obvious to passive viewers (Mack \& Rock, 1998; Most et al., 2001; Neisser, 1979; Simons \& Chabris, 1999). Inattentional blindness is not merely a laboratory phenomenon, but has practical consequences as well; it contributes to medical errors (Lum, Fairbanks, Pennington, \& Zwemer, 2005) and automobile accidents (Strayer \& Drews, 2007).

Despite the range of examples of inattentional blindness in the literature, as well as the apparent pervasiveness of the phenomenon in daily life, few studies have explored whether individual differences contribute to the failure to notice unexpected events. The study of individual differences in inattentional blindness is problematic because the phenomenon is, by definition, limited to one critical trial for each observer (Simons, 2007); once observers are aware that an unexpected object might appear, it no longer is unexpected, and they might voluntarily direct attention to it. Consequently, it is difficult, if not impossible, to measure the reliability of detection across trials for an individual. ${ }^{1}$
To bypass the problem of repeatedly testing the same individual, a few studies have explored individual differences by comparing differences in detection rates across groups of participants. A growing literature has examined individual differences in attention as a function of practice (Boot, Kramer, Simons, Fabiani, \& Gratton, 2008) or of expertise in an attention-demanding task, such as sports (Enns \& Richards, 1997) or video games (Boot et al., 2008; Green \& Bavelier, 2003). Many studies have shown group differences between experts and novices on basic attention measures (e.g., Boot et al., 2008; but see Memmert, Simons, \& Grimme, 2009, for a discussion of some limitations on these advantages for athletes). Training might improve performance on related attention tasks as well, although it is less clear that training necessarily generalizes to measures of attention or perception that are structurally distinct from the domain of expertise (Boot et al., 2008).

Although various forms of expertise or training may enhance performance on some attention-demanding tasks, it is less clear whether such individual or group differences exist for the detection of unexpected objects. Only a few studies have examined group differences in the detection of an unexpected event. In one study (Memmert, 2006), expert basketball players were more likely than experts at an individual sport to notice an unexpected person in a gorilla suit when the primary task involved counting basketball passes. However, expert team handball players were no more likely than individual sport athletes or nonathletes to notice the gorilla (Memmert et al., 2009). Together, these

D. J. Simons, dsimons@illinois.edu 
findings suggest that expertise in a particular sport may benefit detection of unexpected objects in the context of that sport, but that the attentional benefits that accompany team sports expertise in general do not account for this distinction. Although other group differences have been examined (e.g., intoxicated individuals are more likely to miss the unexpected gorilla than are sober individuals; Clifasefi, Takarangi, \& Bergman, 2006) or proposed (e.g., people with autism might be more likely to notice; see Grandin \& Johnson, 2005, p. 25), it is not entirely clear what factors drive such individual differences.

To determine what such group differences tell us about individual differences in inattentional blindness, we must first consider why some people notice an unexpected object whereas others do not. Noticing an unexpected object on a critical trial could be due to random variability, so that any given individual would be equally likely to notice the unexpected object. For example, if $50 \%$ of people noticed the unexpected object during an experiment, the particular $50 \%$ who noticed could be entirely arbitrary -if the test could be repeated, there would be no relationship between the people who noticed the first and the second times. Alternatively, individual differences in some aspect of attention could make some participants more likely than others to notice the unexpected object. Accordingly, if the same participants could be retested, those who missed it the first time would be more likely to miss it the second time. Evidence of group differences in inattentional blindness supports the idea that some aspects of expertise or mental state can affect the detection of unexpected objects, suggesting that individual differences play a role in such detection.

One possible source of individual differences in noticing is facility in performing the primary task. Although a number of studies have examined individual differences in attention performance as well as the relationship between expertise and performance (e.g., Green \& Bavelier, 2003), none of the studies specifically explored the relationship between attention performance and the detection of unexpected events. A few studies, however, have explored how variations in the primary task affect overall noticing rates. For example, when the primary task occurs away from fixation, observers are more likely to miss an unexpected object appearing at fixation (Mack \& Rock, 1998). More importantly for the present studies, increasing the difficulty of the primary task leads to decreased noticing rates with brief static displays (Cartwright-Finch \& Lavie, 2007) and with dynamic monitoring tasks (Simons $\&$ Chabris, 1999). To the extent that task difficulty influences noticing rates, individual differences in the ability to perform the primary task might also produce differences in noticing rates.

This article explores whether individual differences in the ability to perform a primary task predict noticing rates for an unexpected object. Although individual differences in personality or in other cognitive skills might also predict noticing of an unexpected object during an attention-demanding task, our focus will be on the asyet-unexplored contribution of individual differences in primary task performance. Experiment 1 eliminated individual differences in the ability to perform the primary task in order to determine whether task difficulty in the absence of individual differences can predict noticing rates. Experiment 2 examined whether individual differences in the ability to perform the primary task predict the probability of noticing an unexpected object.

\section{EXPERIMENT 1}

\section{Method}

Participants. A total of 46 undergraduate students (24 male, 22 female) participated voluntarily in exchange for credit in an introductory psychology course. All of them reported normal or corrected-to-normal vision. Data from 3 of the participants were discarded because these individuals were familiar with the phenomenon of inattentional blindness.

Materials and Procedure. All displays were presented on a Macintosh eMac computer with a 17 -in. CRT monitor $(1,024 \times$ 768), and the experimental displays and timing were controlled using custom software written in Python using the VisionEgg libraries (described in Straw, Warrant, \& O'Carroll, 2006). The viewing distance was approximately $57 \mathrm{~cm}$ but was not restrained (a distance of $57 \mathrm{~cm}$ is assumed for all visual angle calculations below).

On each trial, participants performed a dynamic tracking task in which they monitored the movements of four white shapes and ignored the movements of four black shapes (see Most et al., 2001, for details). Their task was to count the total number of times that any of the white shapes touched the sides of a $640 \times 480$ pixel gray window $\left(20.3^{\circ} \times 15.2^{\circ}\right)$ centered on the monitor. For each color, two of the shapes were capital Ls $\left(1.27^{\circ} \times 1.27^{\circ}\right)$ and two were capital Ts $\left(1.27^{\circ} \times 1.27^{\circ}\right)$. All objects moved independently on randomly determined trajectories, with the following constraints: (1) Motion was linear between changes of direction; (2) every $10 \mathrm{msec}$, the object turned by a randomly determined amount ranging from $0^{\circ}$ to $5^{\circ}$; (3) whenever an object touched the side of the display window, it rebounded at a randomly chosen angle, with additional constraints to prevent an object from bouncing repeatedly in a corner of the window (see Figure 1).

Each trial lasted approximately $8,200 \mathrm{msec}$, and after each trial, participants were prompted to type the total number of times the white shapes touched the sides of the display. The participants first completed a set of 10 practice trials (without unexpected-object trials) in which the objects moved at a fixed rate of $4.32^{\circ} / \mathrm{sec}$. (All objects on a given trial moved at the same speed.) Following the practice trials, participants completed two blocks of trials in which the speed of the objects in the display was adjusted from trial to trial on the basis of the accuracy of performance (using the Python port of the QUEST algorithm; Watson \& Pelli, 1983). Counting performance was considered accurate when a response was within $20 \%$ of the correct total (rounding up). For example, if the correct count were 9 , any response between 7 and 11 would be counted as correct $(20 \%$ of 9 is 1.8 , which was rounded up to define a range of \pm 2 ). This buffer was introduced in order to avoid penalizing participants too much for slight counting errors when an object bounced repeatedly in a corner. Following an accurate count, the speed of the objects was increased, and following an inaccurate count, it was decreased. In this manner, the object speeds were adjusted for each individual to achieve a consistent level of accuracy across participants. The algorithm was considered to have settled on a threshold speed when the adjusted speeds were within a range of 3 pixels/sec over 10 consecutive trials. All participants completed one QUEST block of trials with the accuracy threshold set to $90 \%$ correct and another with the threshold set to $60 \%$ correct. The order of these blocks was counterbalanced across participants ( 21 had the $90 \%$ block first, and 22 had the $60 \%$ block first). 

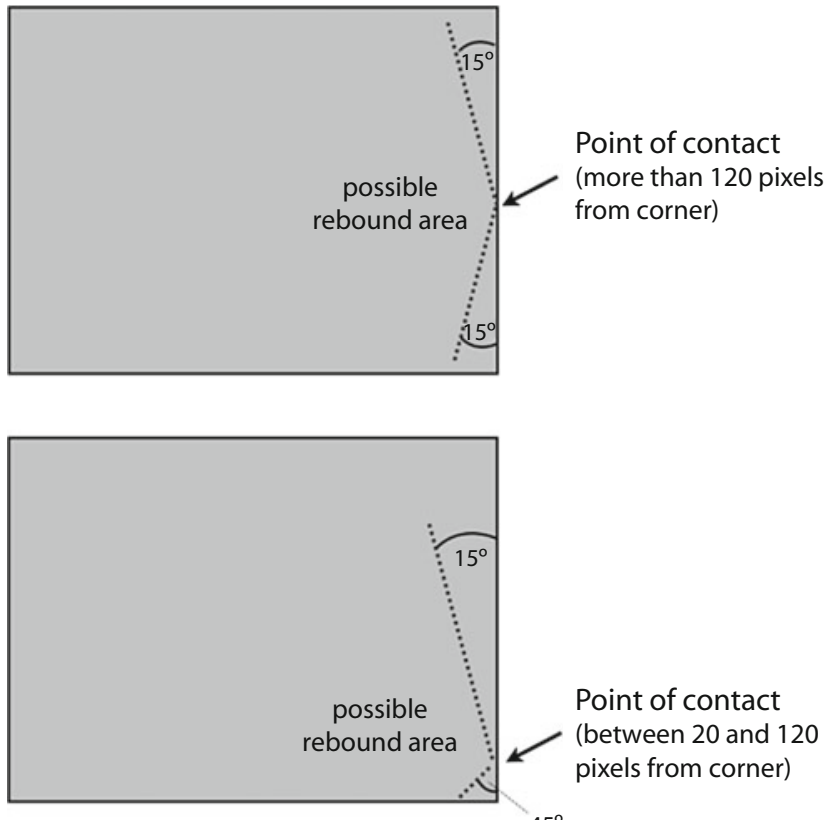

Point of contact

(between 20 and 120

$45^{\circ}$

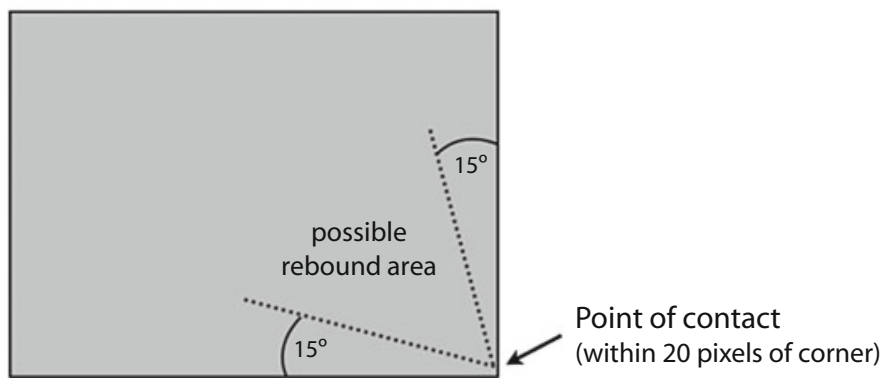

Figure 1. Schematic illustration of the constraints on object rebound trajectories (not to scale). The top panel shows that when the object touched a side of the display more than 3 object widths (120 pixels) away from the corner, a random vector was chosen from a range of $150^{\circ}$, such that after the rebound, the object moved on a trajectory at least $15^{\circ}$ away from the side it had contacted. The middle panel shows that when an object touched the side of the display between 0.5 and 3 object widths (20-120 pixels) from a corner, the rebound angle was constrained to a range of $120^{\circ}$, such that the object moved away from the touched wall by at least $45^{\circ}$ on the side closest to the corner. The bottom panel shows that when the object touched a side within 0.5 object widths ( 20 pixels) of a corner, it rebounded such that it moved away from that corner by at least $\mathbf{1 5}^{\circ}$.

In each block, immediately following the determination of the threshold speed and with no forewarning, observers viewed one critical trial. On this critical trial, after $3 \mathrm{sec}$ of object motion, a dark gray cross $\left(1.27^{\circ} \times 1.27^{\circ}\right)$ entered the display vertically centered on the right, moved linearly from right to left, and exited the display at the vertical midpoint on the left side. The gray cross was visible for $4.7 \mathrm{sec}$, moving at a rate of $4.32 \% \mathrm{sec}$. After the cross exited the display, the objects continued moving for $500 \mathrm{msec}$. After reporting the number of touches, participants were first asked "Did you notice anything other than the Ls and Ts on that last trial?" and then asked to "Describe what you saw." For these two questions, each individual typed the responses into a text field on the display.

Each participant completed both a $60 \%$ and a $90 \%$ block to verify that the manipulation of the threshold had an effect on tracking speed within an individual. However, for the critical trial following the second QUEST block, the gray cross was no longer entirely unexpected (since participants had already been asked about it following the first block). For this reason, the primary analyses are based on performance on the critical trial during the first QUEST block for a given participant, and comparisons of the effects of tracking accuracy on noticing of the unexpected object were conducted across groups of participants.

\section{Results and Discussion}

The number of trials required to determine the threshold speed varied across participants, although the average numbers of trials were comparable across groups (see Table 1). Overall, $51.2 \%$ of the participants failed to report the gray cross. The $90 \%$ threshold condition required more accuracy in performance, meaning that the tracking 
Table 1

Average Number of Trials (and Standard Deviation) Needed to Reach Threshold, Average Object Speed (and Standard Deviation) at Threshold, and Percentage Noticing the Unexpected Object, As a Function of the Achieved Level of Tracking Accuracy

\begin{tabular}{|c|c|c|c|c|}
\hline & \multicolumn{2}{|c|}{$\begin{array}{c}60 \% \text { Tracking } \\
\text { Accuracy }\end{array}$} & \multicolumn{2}{|c|}{$\begin{array}{c}90 \% \text { Tracking } \\
\text { Accuracy }\end{array}$} \\
\hline & $M$ & $S D$ & $M$ & $S D$ \\
\hline Number of trials until threshold & 37.64 & 10.25 & 32.67 & 8.72 \\
\hline Tracking speed at threshold (deg/sec) & 4.63 & 0.839 & 3.65 & 0.531 \\
\hline Percent detecting unexpected object & 31.8 & & 71.4 & \\
\hline
\end{tabular}

task was less demanding (i.e., the objects moved slower). Consistent with the idea that the tracking task was easier in the $90 \%$ than in the $60 \%$ threshold condition, participants in the $90 \%$ condition were more likely to notice the cross $\left[71.4 \%\right.$ vs. $31.8 \% ; \chi^{2}(1, N=21 / 22)=6.747, p=$ .0094]; more-difficult tasks lead to less noticing of unexpected objects (Cartwright-Finch \& Lavie, 2007; Simons \& Chabris, 1999). Critically, the experiment demonstrated an effect of task difficulty that occurred independently of any individual differences in tracking ability, since the difficulty of the task was calibrated separately for each participant. $^{2}$

In this experiment, the unexpected object moved at a speed $(4.32 \% \mathrm{sec})$ intermediate to the average threshold tracking speeds for the hard $(4.63 \% \mathrm{sec})$ and easy $\left(3.65^{\circ} \%\right.$ $\mathrm{sec})$ conditions. One concern is that the relative difference between the unexpected object speed and the average threshold tracking speeds might contribute to the difference in noticing between the conditions. If so, the deviation between a participant's threshold tracking speed and the critical object speed should correlate with the probability of noticing the unexpected object. The pattern was inconsistent with respect to this alternative explanation $(r=.0665)$. Even if we factor out the overall difference in tracking speeds between the easy and hard conditions by standardizing these deviations within a condition and correlating the standardized scores with noticing, noticing is not significantly correlated with the similarity of the unexpected object speed to the speed of the other objects in the display $(r=.08)$. This lack of an effect of similarity in speed is consistent with work showing that variation along unattended dimensions has relatively little impact on noticing (Most, Scholl, Clifford, \& Simons, 2005), because in our study speed did not distinguish targets from distractors.

\section{EXPERIMENT 2}

Experiment 1 showed that task difficulty directly affects the probability of noticing an unexpected object when individual differences in primary task performance are eliminated. Experiment 2 explored whether individual differences in tracking ability predict noticing rates. Given that increased task difficulty leads to less noticing, individuals who are better at the primary task might be more likely to notice the unexpected object-for them, the primary task requires fewer resources. Alternatively, the probability of noticing might not vary with the task difficulty for a given individual. For this alternative hypothesis to be true, accuracy on the primary task should not predict noticing.

In line with the counterintuitive idea that primary task performance does not predict noticing, accuracy of counting in a video-based inattentional blindness task was only weakly correlated ( $r=.15$ across conditions) with noticing the unexpected object (Simons \& Chabris, 1999). Likewise, in a computer-based inattentional blindness task, the accuracy of counting on trials prior to the critical trial was unrelated to noticing the unexpected object (Most et al., 2005). ${ }^{3}$ These findings suggest that as long as participants try equally hard to do the task, noticing rates are driven by the actual task difficulty rather than by the ability to perform the task accurately. That is, participants may try equally hard to do a task, with some achieving better performance than others, but the level of performance does not predict noticing of an unexpected object.

\section{Method}

Participants. A total of 109 undergraduate students participated in exchange for credit in an introductory psychology course. All reported normal or corrected-to-normal vision. The data from 27 individuals were discarded, for the following reasons: familiarity with the phenomenon of inattentional blindness $(n=14)$, failure to follow instructions $(n=7)$, computer error $(n=3)$, or experimenter error $(n=3)$. These exclusions left 82 participants (46 male, 36 female) for the analysis. A larger number of participants were included in this experiment in order to increase the power to find individual differences as a function of tracking speed.

Task and Procedure. Except as noted, the procedure was identical to that of Experiment 1. All participants first completed 10 practice trials with all objects moving at $4.32 \% \mathrm{sec}$. They then completed two blocks of trials that adaptively determined the object speed necessary for $75 \%$-accurate tracking performance (using QUEST). Threshold tracking speeds on the two blocks were averaged to produce a more stable measure of tracking ability (i.e., how fast a participant could track the objects and still achieve $75 \%$ accuracy). Neither the practice block nor the QUEST blocks included any unexpected objects. Following these blocks, all participants completed 10 trials at a fixed speed $(4.32 \% \mathrm{sec})$, followed by 1 critical trial at the same speed in which an unexpected object moved across the display (also at $4.32 \% \mathrm{sec}$ ). The speeds in this final block of trials and in the critical trial were constant across participants to provide a stable measure of inattentional blindness.

\section{Results and Discussion}

Overall, $65.9 \%$ of the participants (54 of 82 ) noticed the unexpected gray cross. Threshold-tracking speeds varied substantially across individuals $\left(M=4.40^{\circ} / \mathrm{sec}\right.$, $S D=0.684$, range $=2.84$ to $6.96^{\circ} / \mathrm{sec}$ ). Strikingly, individual differences in tracking speed were not correlated with noticing of the unexpected object $(r=.099, p=$ .372; mean tracking speed for noticers $=4.44^{\circ} / \mathrm{sec}$, mean tracking speed for nonnoticers $\left.=4.30^{\circ} / \mathrm{sec}\right)$. Even when we compared the fastest $20 \%$ of trackers $(n=16, M=$ $5.41 \% \mathrm{sec}, S D=0.554)$ with the slowest $20 \%$ of trackers $(n=16, M=3.56 \% \mathrm{sec}, S D=0.317)$, noticing rates ( $75 \%$ and $62.5 \%$, respectively) did not differ significantly $\left[\chi^{2}(1)=0.582, p=.446\right]$, even though the groups did not overlap at all in terms of tracking speeds. Apparently, individual differences in the speed at which people can 
track objects accurately do not predict the detection of unexpected objects.

An alternative explanation for the lack of correlation between tracking speeds and noticing is that there was insufficient variability to detect a correlation. Noticing rates in this experiment were far from the $100 \%$ detection ceiling, suggesting that there was adequate room to show a difference between good and poor trackers. However, the tracking speed used on the critical trial might have been too easy for most participants, limiting the variability in tracking speeds. To examine this possibility, we correlated each person's $75 \%$ threshold with the number of practice trials for which they tracked accurately $(M=6.04$ out of $10, S D=2.14$, range $=1-10$ correct out of 10 ; the practice trials used the same speed as the critical trial, $4.32 \%$ sec).$^{4}$ If the fixed speed on the critical trial were too easy, leading to a ceiling effect, little or no correlation should appear between tracking for the $75 \%$ threshold and accuracy during the practice trials (i.e., there would be insufficient variability in tracking performance because the task was easy for everyone). Yet, the correlation between tracking accuracy at the fixed speed and the threshold tracking speed was $r=.538$; people who were able to track at a faster speed were more accurate at the fixed speed, showing that there was sufficient variability to detect a relationship between tracking accuracy and noticing. Thus, the lack of a correlation between threshold tracking speed and noticing of the unexpected object suggests that individual differences in tracking performance are largely unrelated to the likelihood of noticing an unexpected object.

\section{GENERAL DISCUSSION}

Taken together, the results of these two experiments suggest that the demands of the primary task affect inattentional blindness rates, but individual differences in the ability to meet those demands do not. In Experiment 1, when performance of the primary task was equated across individuals by adjusting the speed of the objects so that participants could perform the primary task equally well, the difficulty of the task predicted noticing rates. However, in Experiment 2, individual differences in the object speed necessary to achieve a given level of tracking performance did not predict noticing of the unexpected object.

Although previous studies have explored the effect of task difficulty on noticing rates in inattentional blindness (Cartwright-Finch \& Lavie, 2007; Simons \& Chabris, 1999), none have systematically eliminated individual differences in primary task performance to do so, and none have examined the effect of individual differences in primary task performance on noticing rates. Our experiments revealed, somewhat counterintuitively, that individual differences in the ability to complete a primary task may play a minimal role in the noticing of unexpected objects. As long as participants try equally hard to do the task, their ability to do it well does not predict noticing rates.

These findings have potentially important ramifications for the practical consequences of inattentional blindness.
Better performance on the primary task did not predict noticing of an unexpected object, raising the possibility that training on the primary task would not improve detection of unexpected objects either. Training likely would improve performance on the primary task, just as training on video games leads to improved performance on those games and on some closely related tasks (Boot et al., 2008; Green \& Bavelier, 2003). However, attentionrelated training may not improve the chances of noticing an unexpected object, even if it does lead to improvements on other attention tasks. Noticing appears to be based on the relative difficulty of the task itself, rather than on differences in how well people can perform the task.

Although individual differences in the ability to perform the primary task do not correlate with noticing rates, other individual differences might well affect noticing, particularly individual differences that influence how hard people try to do the task. For example, individual differences in conscientiousness or other personality factors might predict noticing, not because they lead to better performance on the task, but because they influence how committed people are to trying to do well. Increasing the task difficulty apparently leads all participants to try harder when doing the task, thereby decreasing noticing rates. Individual differences in distractibility or in other factors unrelated to primary task performance also might contribute to noticing. Further research is needed to explore other individual-difference variables that could contribute to inattentional blindness, as well as to verify whether or not training on the primary task (or on related attention tasks) could decrease rates of inattentional blindness.

In sum, these are the first inattentional blindness experiments to control the difficulty of the primary task across individuals and to explore how individual differences in the ability to perform the primary task influence noticing of an unexpected object. Although other studies have varied the difficulty of the primary task, none have systematically measured the ability to perform the primary task prior to the critical trial containing the unexpected object. Consistent with earlier evidence, our study shows that task difficulty influences the rate of inattentional blindness. Surprisingly, though, the accuracy with which people performed the primary task was unrelated to noticing of the unexpected object. Together, these findings show that inattentional blindness is driven by the difficulty of the primary task, not by performance on that task.

\section{AUTHOR NOTE}

Both authors contributed to the original ideas for the experiments. D.J.S. wrote the first draft of the manuscript, oversaw the design and implementation of the study, and contributed to the data analysis and the revision of the manuscript. M.S.J. conducted the study and contributed to the data analysis and revision of the manuscript. We thank Michael Almaraz and Rafael Fernandez for programming assistance and Anne Rufa, Mandy Wolf, and Katie Fuechsl for help with data collection. Thanks also to Ben Tatler, Peter McCormick, and an anonymous reviewer for helpful suggestions. Correspondence related to this article may be sent to D. J. Simons, Department of Psychology, University of Illinois, 603 E. Daniel Street, Champaign, IL 61822 (e-mail: dsimons@ illinois.edu). 


\section{REFERENCES}

Boot, W. R., Kramer, A. F., Simons, D. J., Fabiani, M., \& Gratton, G. (2008). The effects of video game playing on attention, memory, and executive control. Acta Psychologica, 129, 387-398.

Cartwright-Finch, U., \& Lavie, N. (2007). The role of perceptual load in inattentional blindness. Cognition, 102, 321-340.

Cliffasefi, S. L., Takarangi, M. K. T., \& Bergman, J. S. (2006). Blind drunk: The effects of alcohol on inattentional blindness. Applied Cognitive Psychology, 20, 697-704.

EnNs, J. T., \& Richards, J. C. (1997). Visual attentional orienting in developing hockey players. Journal of Experimental Child Psychology, 64, 255-275.

Grandin, T., \& Johnson, C. (2005). Animals in translation: Using the mysteries of autism to decode animal behavior. New York: Simon \& Schuster.

Green, C. S., \& Bavelier, D. (2003). Action video game modifies visual selective attention. Nature, 423, 534-537.

Kunn, G., Amlani, A. A., \& Rensink, R. A. (2008). Towards a science of magic. Trends in Cognitive Sciences, 12, 349-354.

Lum, T. E., Fairbanks, R. J., Pennington, E. C., \& Zwemer, F. L. (2005). Profiles in patient safety: Misplaced femoral line guidewire and multiple failures to detect the foreign body on chest radiography. Academic Emergency Medicine, 12, 658-662.

MACK, A., \& RocK, I. (1998). Inattentional blindness. Cambridge, MA: MIT Press.

Macknik, S. L., King, M., Randi, J., Robbins, A., Teller, ThompSON, J., \& MARTINEZ-Conde, S. (2008). Attention and awareness in stage magic: Turning tricks into research. Nature Reviews Neuroscience, 9, 871-879.

Memmert, D. (2006). The effects of eye movements, age, and expertise on inattentional blindness. Consciousness \& Cognition, 15, 620-627.

Memmert, D., Simons, D. J., \& Grimme, T. (2009). The relationship between visual attention and expertise in sports. Psychology of Sport \& Exercise, 10, 146-151.

Most, S. B., Scholl, B. J., Clifford, E. R., \& Simons, D. J. (2005). What you see is what you set: Sustained inattentional blindness and the capture of awareness. Psychological Review, 112, 217-242.

Most, S. B., Simons, D. J., Scholl, B. J., Jimenez, R., Clifford, E., \& Chabris, C. F. (2001). How not to be seen: The contribution of similarity and selective ignoring to sustained inattentional blindness. Psychological Science, 12, 9-17.

NeISSER, U. (1979). The control of information pickup in selective looking. In A. D. Pick (Ed.), Perception and its development: A tribute to Eleanor J. Gibson (pp. 201-219). Hillsdale, NJ: Erlbaum.

Simons, D. J. (2007). Inattentional blindness. Scholarpedia, 2, 3244. Available at www.scholarpedia.org/article/Inattentional_blindness.

Simons, D. J., \& Chabris, C. F. (1999). Gorillas in our midst: Sus- tained inattentional blindness for dynamic events. Perception, 28, 1059-1074.

Straw, A. D., Warrant, E. J., \& O'Carroll, D. C. (2006). A “bright zone" in male hoverfly (Eristalis tenax) eyes and associated faster motion detection and increased contrast sensitivity. Journal of Experimental Biology, 209, 4339-4354.

Strayer, D. L., \& Drews, F. A. (2007). Cell-phone-induced driver distraction. Current Directions in Psychological Science, 16, 128-131.

Watson, A. B., \& Pelli, D. G. (1983). QUEST: A Bayesian adaptive psychometric method. Perception \& Psychophysics, 33, 113-120.

\section{NOTES}

1. One possible exception to the rule that people can only be fooled once in an inattentional blindness task comes from stage magic. Magicians are able to produce the same unexpected outcomes repeatedly. For example, they can make a set of balls disappear without observers detecting how they did so. Note, though, that magicians rarely do exactly the same trick in exactly the same way twice in a row, since that would make it more likely that observers would detect their method. In some respects, the tendency to be fooled by magic tricks is more akin to change blindness (see Kuhn, Amlani, \& Rensink, 2008; Macknik et al., 2008), in that people fail to see how something came about rather than failing to notice the end state. That is, they fail to notice how a bunny suddenly appeared in the magician's hand, but they do see the bunny.

2. Not surprisingly, noticing rates were higher in the second block, when the critical object was no longer completely unexpected (in the $60 \%$ condition, $63.64 \%$ detection, $4.93 \% \mathrm{sec}$ tracking; in the $90 \%$ condition, $85.71 \%$ detection, $3.67^{\circ} / \mathrm{sec}$ tracking). Overall tracking speeds did not differ across blocks $[t(42)=0.657, p=.514]$. The pattern of greater detection in the $90 \%$ condition was maintained across blocks, although the difference was somewhat attenuated relative to the first block, in which noticing rates were lower $\left[\chi^{2}(1, N=21\right.$ and 22$\left.)=2.751, p=.097\right]$.

3. Note that Most et al. (2005) did find an effect of accuracy on the critical trial itself, with both noticers and nonnoticers showing greater counting errors, but noticers showing worse accuracy than did nonnoticers. Although interesting, changes to the error rates on the critical trial do not speak to the question of whether the ability to perform the primary task is related to noticing. It is possible that the presence of the unexpected object led to changes in counting performance.

4. Because of a programming glitch, tracking accuracy data were not collected on the 10 critical trials at the end of the experiment. Those trials used the same speed as the practice trials, though, so here we have used the practice trials to test the idea that there was insufficient variability to detect a correlation.

(Manuscript received May 20, 2008; revision accepted for publication November 20, 2008.) 